\title{
REVIEW AND DEBATE: Hardwood fibre pits - Again!
}

Morphology of pits in hardwood fibres. Part I by J.F.C. Magendans, Part II by J.F.C. Magendans \& W.L.H. van Veenendaal, 100 pp., illus., 1999. Wageningen Agricultural University Papers 99-2. Backhuys Publishers (P. O. Box 321, 2300 AH Leiden, The Netherlands; e-mail: backhuys@euronet.nl). ISBN 90-5782-036-6. Price: NLG 52 (paperback).

These two papers combine a micromorphological study of pits in hardwood fibres with an in-depth discussion of their distinction from bordered pits. In the first part Dr. Magendans provides a very detailed three-dimensional analysis of fibre pits in Alnus viridis and Solanum lycopersicum. Fibres in these species all have funnel (or calyciform) pits, that differ from bordered pits in their gradual rather than abrupt widening of the pit canal towards the middle lamella, and in that the widest part of the pit cavity never fully fits that of the complementary half of the pit pair. Or in other words the outer pit apertures of the complementary pit canals have more or less shifted positions in relation to each other. Intervessel pits in contrast have a well-defined lenticular pit cavity, and the complementary parts of a pit pair are fully matching. These very large differences are considered fundamental, and a case is made for the homology of fibres with sclerenchyma and parenchyma cells where funnel pits also have been recorded. Arguments from physiology and xylem development and comparative morphology are invoked to support this view.

In the second paper the study of fibre pits is extended to 15 other species from 14 different families and with greatly differing sizes of the funnel rims (i.e., what used to be called the pit borders in traditional descriptive accounts), ranging from less than 2 to over 6 microns. The point is made that in all species the diameter of these funnel rims are significantly smaller than that of the borders of the intervessel pits. According to Magendans and Van Veenendaal all these species show funnel pits with funnel margins shifted with respect to the complementary halves of the pit pair. Despite the large size of the funnel rims in Anthocephalus and Nyssa, and the rather well-defined lenticular shape of the pit cavities in sectional view (Fig. 26 and 42-46), the authors consider these fibre pits to be fundamentally different from bordered pits and the fibres to be of the libriform type.

Throughout, the authors challenge the traditional interpretation of the diversity in imperforate tracheary cell wall pitting as a continuum from fully bordered in tracheids to minutely bordered and simple pits in libriform fibres. This is partly because within none of the species they studied such a continuum was observed, and partly because once narrowly defined (on pp. 36-37) funnel pits and bordered pits remain different 'by definition'. However, in my opinion, even that difference (based incidentally on circular argument) breaks down in the examples of Anthocephalus and Nyssa (and many other hardwoods with fibre-tracheids or 'true' tracheids sensu Carlquist), where the pit cavity is as lenticular as in any intertracheid or intervessel pit pair, but where 
the shifted positions of the pit cavities are the only criterion that fit the definition of the funnel pits. I suspect that those shifted positions are caused by the fact that fibres as opposed to tracheids and vessel elements show elongation by intrusive and even sliding growth before their differentiation and pit formation is completed, but I cannot find those positional shifts of the pit cavity or the subtle shape differences of the pit cavity 'fundamental'. They are certainly highly interesting from a developmental point of view and worthy of study. Yet Magendans claims that a morphological continuum between tracheids and fibres "surely does not exist at all." Two other reasons given by Magendans on p. 24 to reject the continuum (and evolutionary transformation) theory dating back to Bailey and Tupper (1918) are that he considers it unlikely that sclerenchyma tissue present near water conducting cells in earlier land plants (mosses, ferns) had to be reinvented again in the secondary xylem of angiosperm wood, and that physiological phenomena indicate the close relationships between sclerenchyma (including libriform fibres) and parenchyma.

To me it seems a pity, that in demonstrating the continuum from funnel pits to simple pits in libriform hardwood fibres and certain parenchyma and sclerenchyma cells, the authors put so much energy in disputing the continuum from bordered pits to funnel pits. That continuum is not to be sought within a single wood specimen but within phylogenetic lineages that represent evolutionary history. Much published 'classical' evidence as well as the most recent molecular phylogenies indicate that the angiosperms were primitively vesselless (Amborella is now firmly at the root of all multiple gene trees of the angiosperms as presented during the XVI International Botanical Congress in St. Louis), i.e., had wood with only tracheids and no vessels or libriform fibres for conduction and support. It requires the most unlikely saltatory evolution to directly evolve a system with vessel elements and libriform fibres with funnel pits from that ancestral type. The close statistical association of fibres with distinctly bordered pits and relatively little intrusive growth with primitive vessel elements also pleads against that, as does the decrease in percentage of hardwoods with distinctly bordered fibre pits (or should I say widely rimmed funnel pits) in the fossil record from the Cretaceous onwards.

We owe the authors a detailed look at fibre pit morphology, and a provocative alternative interpretation of hardwood fibre homology. I find the arguments for that alternative homology unconvincing and wholly unnecessary. After all, all fusiform cambial elements can be viewed as homologous, and to me the additional morphological information presented supports the complete continuum in angiosperms between tracheids, vessel elements, fibre-tracheids, libriform fibres and axial parenchyma. That continuum can only be interpreted in an evolutionary sense within individual lineages of the angiosperms in the presence of independent data on their phylogeny.

Pieter Baas 


\section{Response to Baas' review of my articles in 'Morphology of pits in hardwood fibres'}

First of all I want to express my appreciation for the attention given by Baas to my articles. In this he has tried to sustain his completely different opinion, viz. all fusiform cambial elements in angiosperms and gymnosperms (and derivatives) can be viewed as homologous, thus forming a complete continuum in angiosperms between tracheids, vessel elements, fibre-tracheids, libriform fibres, axial parenchyma and all axial elements in the phloem (Baas, in Terminology of imperforate tracheary elements, 1986). As vessel elements and sieve elements have structurally nothing in common, not to mention transitional forms, this is only possible when comparative morphology as an important scientific discipline is virtually completely rendered inoperative. The very sophisticated and uniform structure of a bordered pit is so specialized that it cannot be compared with the variable form of a simple pit. It is really necessary to emphasize the significance of comparative morphology in distinguishing important differences in structure and thus it will remain important to observe microscopic structures minutely. For example the descriptive convention of the $3 \mu \mathrm{m}$ limit (IAWA List of microscopic features for hardwood identification, 1989) of the diameter of 'borders' cannot be applied by morphologists. This measure cannot be assessed in practice because of the shifted positions of outer apertures of complementary pit canals in relation to each other along the middle lamella. The characteristic of being 'co-ordinated' belongs to bordered pits and forms a developmental continuum with the wall strengthening structures in tracheary elements of fossil plants. While sieve areas in the overpressured sieve elements are homologous with simple pits, the bordered pits in the underpressured tracheary elements originated between and from the original, essential strengthening rings within the vessel and tracheid primordia. Thus the completely different evolutionary development of bordered pits in tracheary elements precludes homology with sieve areas and simple pits; the rather complex structure of a bordered pit is consequently closely connected with the evolutionary development of tracheary elements. Homologous, at this level of wall structures, means the same general construction and corresponding positioning, while transitional forms and the same specific evolutionary origin and development are characteristic. As morphologist I do consider it as my task drawing attention to differences in form, how small they may be, and in case of consistent appearance trying to compare these results with other data, also from other disciplines. For example to find a different functional or ontogenetic specialisation, and thus trying to discover rather fundamental differences in structures and formulate definitions of them to make these structural differences reproducible. In my opinion there is no circular argument in this operating procedure. For example the positional shifting of outer apertures of the complementary pit canals in funnel pits (p. 37), always going together with more characteristic differences with bordered pits as the non-congruency of the pit halves, is considered by Baas as a bagatelle. He considers the pits in Fig. 25 (Anthocephalus, p. 68) and 41 (Nyssa, p. 78) as examples of real bordered pits, an opinion that is firmly rejected by me as morphologist. Besides, it must be remembered that the exact coordination of wall strengthening structures in the primitive conductive elements in the xylem of Völkelia refracta (Mid Devonian period, Henes, in: Fossile Wandstrukturen, 1959) shows the early importance of an accurate coordination. Also Esau (in: Plant Anatomy, 1965, Fig. 
11.4) shows the perfect coordination between helical wall strengthening structures of adjacent tracheary elements, essential precursor-positioning for the later developing bordered pits. The suggestion of (intrusive) growth as a main factor in causing shifting of outer pit apertures in fibre pits is probably wrong, because adjacent tracheary elements in vein endings show also perfect coordination of spiral strengthening structures though showing an intense intrusive growth of the procambial precursor cells.

Baas argues that the presumed continuum between simple pits and bordered pits is not to be sought within a single wood specimen but within phylogenetic lineages that represent evolutionary history. I think that definite examples of intermediary forms between structures, elements and tissues within a single wood specimen should be evident in hardwoods, for demonstrating the continuum hypothesis. And in my opinion it is not allowed to construct (in mind) a series of intermediary structures out of many wood species without direct relation with each other. In that case the produced 'continuum' of pits is based on circular argument.

Amborella may now be firmly at the root of all multiple gene trees of the angiosperms and may show only tracheids as axial elements and no vessels or libriform fibres for conduction and support. But, parenchyma and/or sclerenchyma are always present in (early) representatives of the plant world and that means that libriform is also potentially present, and thus a "most unlikely saltatory evolution to directly evolve a system with vessel elements and libriform fibres" is certainly not required.

In summary Baas ignores comparative morphology as an authentic discipline; why he does so is, to me, obscure. Then I must repeat: there is, from a morphological but also from a physiological point of view, no reason to assume the existence of a morphological continuum between simple and funnel pits on the one hand and bordered pits on the other. And as long as agreement about evolutionary development of fibres is not established, comparative morphology remains essential.

N.B. Simple pits and sieve areas: places of cell contact within symplast systems

Funnel pits: idem, but often shorter-lived (e.g. fibres)

Bordered pits: places of cell contact within apoplast systems.

J.F.C. Magendans

\section{Comments on Magendans' response and call to join the debate}

Readers are invited to join the above debate on morphology and homology of plant cell types. At this stage I only want to take away some misunderstandings that might arise from Magendans's interpretation of my review. 1) Although in a very broad sense, phloem and xylem derivatives can all be homologised, I think it is defensible to adopt a narrower homology concept and restrict it to the xylem and phloem elements separately; 2) I fully admit that the 3 micron limit in pit border (or funnel rims) of fibre pits is arbitrary - the fact that the IAWA Committee resorted to this arbitrary measure testifies to the continuum present in hardwood fibre pit (border or funnel rim) diameters; 3) Like all wood anatomists I fully subscribe to Magendans's view that comparative morphology is essential for all our work. On that very basis I cannot possibly ignore the fully bordered nature of the fibre pits in Anthocephalus, Nyssa, and many other hardwoods. Perhaps our difference is that between typological and evolutionary comparative morphology. 